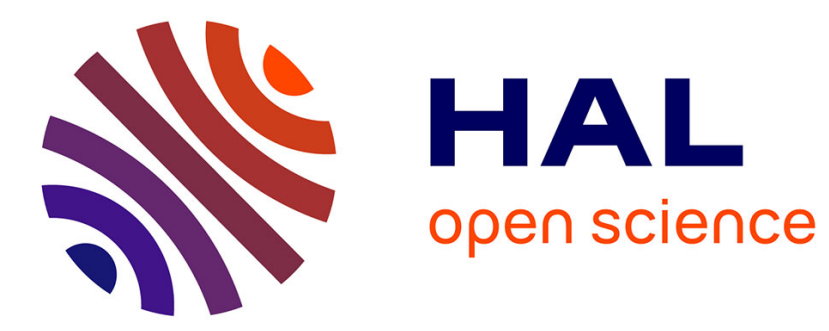

\title{
Unprecedented Observation of Days-Long Remnant Orientation of Phospholipid Bicelles: A Small-Angle X-ray Scattering and Theoretical Study
}

\author{
Cecile Courreges, Frédéric Nallet, Erick J. Dufourc, Reiko Oda
}

\section{- To cite this version:}

Cecile Courreges, Frédéric Nallet, Erick J. Dufourc, Reiko Oda. Unprecedented Observation of DaysLong Remnant Orientation of Phospholipid Bicelles: A Small-Angle X-ray Scattering and Theoretical Study. Langmuir, 2011, 27 (15), pp. 9122-9130. 10.1021/la1050817 . hal-00616617

\section{HAL Id: hal-00616617 https://hal.science/hal-00616617}

Submitted on 5 Oct 2017

HAL is a multi-disciplinary open access archive for the deposit and dissemination of scientific research documents, whether they are published or not. The documents may come from teaching and research institutions in France or abroad, or from public or private research centers.
L'archive ouverte pluridisciplinaire $\mathbf{H A L}$, est destinée au dépôt et à la diffusion de documents scientifiques de niveau recherche, publiés ou non, émanant des établissements d'enseignement et de recherche français ou étrangers, des laboratoires publics ou privés. 


\title{
Unprecedented Observation of Days-Long Remnant Orientation of Phospholipid Bicelles: A Small-Angle X-ray Scattering and Theoretical Study
}

\author{
Cécile Loudet-Courreges, ${ }^{+, \S}$ Frédéric Nallet, ${ }^{\ddagger}$ Erick J. Dufourc, ${ }^{\dagger}$ and Reiko Oda ${ }^{+, *}$ \\ ${ }^{+}$UMR 5248 CBMN, CNRS-Université Bordeaux1-ENITAB, Institut Européen de Chimie et Biologie, Pessac, France \\ ${ }^{\ddagger}$ Université Bordeaux-1, centre de recherche Paul-Pascal - CNRS, Pessac, France \\ ${ }_{\S}^{\S}$ Present affiliation, Institut Européen de Chimie et Biologie UMS3033, Pessac, France
}

\begin{abstract}
Nanometric bilayer-based self-assembled micelles commonly named as bicelles, formed with a mixture of long and short chains phosphatidylcholine lipids (PC), are known to orient spontaneously in a magnetic field. This field-induced orientational order strongly depends on the molecular structure of the phospholipids. Using small-angle X-ray scattering (SAXS), we performed detailed structural studies of bicelles and investigated the orientation/relaxation kinetics in three different systems: saturated-chain lipid bicelles made of DMPC (dimyristoyl PC)/DCPC (1,2-dicaproyl PC) with and without the added paramagnetic lanthanide ions $\mathrm{Eu}^{3+}$, as well as bicelles of TBBPC (1-tetradecanoyl2-(4-(4-biphenyl)butanoyl)-sn-glycero-3-PC)/DCPC. The structural study confirmed the previous NMR studies, which showed that DMPC bicelles orient with the membrane normal perpendicular (defined here as "nematic" orientation) to the magnetic field, whereas they orient parallel (defined here as "smectic"

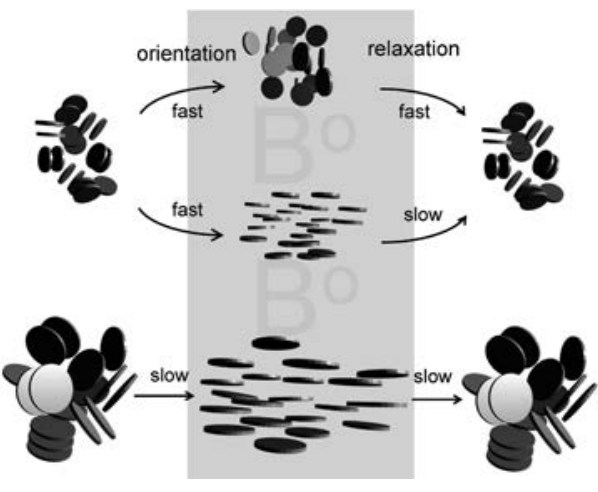
orientation) to the magnetic field in the presence of $\mathrm{Eu}^{3+}$. The TBBPC bicelles also show smectic orientation. Surprisingly, the orientational order induced in the magnetic field remains even after the magnetic field is removed, which allowed us to investigate the orientation and relaxation kinetics of different bicelle structures. We demonstrate that this kinetics is very different for all three types of bicelles at the same lipid concentration; DMPC bicelles $(\sim 40 \mathrm{~nm}$ diameter $)$ with and without Eu ${ }^{3+}$ orient faster than TBBPC bicelles ( $\sim 80 \mathrm{~nm}$ diameter). However, for the relaxation, DMPC bicelles (nematic) lose their macroscopic orientation only after one hour, whereas both DMPC bicelles with $\mathrm{Eu}^{3+}$ and TBBPC bicelles (smectic) remarkably stay oriented for up to several days! These results indicate that the orientation mechanism of these nanometric disks in the magnetic field is governed by their size, with smaller bicelles orienting faster than the larger bicelles. Their relaxation mechanism outside the magnetic field, however, is governed by the degree of ordering. Indeed, the angular distribution of oriented bicelles is much narrower for the bicelles with smectic orientation, and, consequently, they keep aligned for much longer time (days) than those with nematic ordering (hours) outside the magnetic field. The understanding of the orientation/relaxation kinetics, as well as the morphologies of these "molecular goniometers" at molecular and supramolecular levels, allows controlling such an unprecedented long-range and long-lived smectic ordering of nanodisks and opens a wide field of applications for structural biology or material sciences.
\end{abstract}

\section{INTRODUCTION}

The amphiphilic property of lipid molecules leads to very rich polymorphisms of their assemblies. Whereas the spherical or cylindrical micelles, or 2D films with mono- or bilayer structures, are the most common, mixtures of two or more lipids with highly different characteristics or the use of catanionic surfactants can sometimes lead to more exotic morphologies such as nanodiscs, icosahedra, or punctured planes. ${ }^{1}$ Bilayer micelles (bicelles) are one of these structures introduced in mid-1980s formed when mixing long-chain (14-18 carbons) and short-chain (6-8 carbons) phospholipids. ${ }^{2,3}$ Under certain temperatures, hydration, and lipid ratios, such mixtures were reported to form bilayer membrane disks with 30-80 nm diameter and about $4 \mathrm{~nm}$ thickness (the bilayer thickness). Because of the nonzero anisotropy of the diamagnetic susceptibility of dialkanoylphospholipids in the whole edifice, $\Delta \chi$ $\left(\Delta \chi=\chi_{\|}-\chi_{\perp}\right.$, where $\chi_{\|}$and $\chi_{\perp}$ respectively represent the magnetic susceptibility parallel and perpendicular to the long lipid axis), bicelles are aligned by high magnetic fields. This property is of considerable interest because it provides scientists interested in structure, topology, and dynamics of membrane proteins versatile biomimetic membranes with the fantastic advantage of controlling hydration, $\mathrm{pH}$, salt content, and temperature and a number of investigations were reported using $\mathrm{NMR}^{4-7} \mathrm{EPR}^{8}$ or SAXS., ${ }^{9,10}$

In the case of most often studied saturated-chain phospholipids, 1,2-dimyristoyl-sn-glycero-3-phosphocholine (DMPC)/ 1,2-dicaproyl-sn-glycero-3-phosphocholine (6:0/6:0) (DCPC) mixtures, $\Delta \chi$ is negative, the membrane normal, $\mathbf{n}$, to the bilayer disk therefore orients perpendicular to the field direction $B_{0}$ 


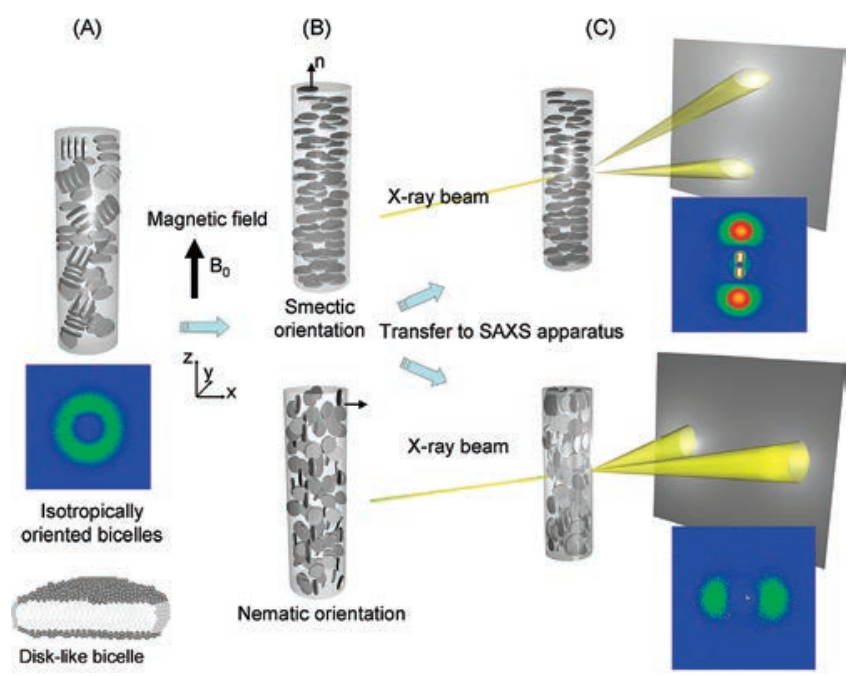

Figure 1. Scheme and corresponding SAXS scattering pattern showing the orientation of DMPC and TBBPC bicelles placed in A) a capillary tube before orientation, B) after orientation for $15 \mathrm{~min}$ in a $11.7 \mathrm{~T}$ magnetic field $\left(B_{0}\right) C$ ) after their transfer in the small-angle X-ray scattering apparatus. The temperature for the whole experiment is set up to $35^{\circ} \mathrm{C}$. The insert shows the molecular organization inside a bicelle.

(referred to as nematic orientation in part a of Figure 1). However, it has been reported that in the presence of species with highly positive diamagnetic susceptibility such as lanthanide ions $\left(\mathrm{Eu}^{3+}\right.$, $\mathrm{Tm}^{3+}$, or $\left.\mathrm{Yb}^{3+}\right)$, which associate with the lipid bilayer surface, the bicelles can flip their orientation by $90^{\circ}$, and the bilayer normal, $\mathbf{n}$, will become parallel to the field direction $B_{0}$ (referred to as smectic orientation in part a of Figure 1). ${ }^{11,8}$

More recently, another type of bicelles was reported, ${ }^{12-16}$ in which DMPC is replaced by modified phospholipids, dodecanoyl-2-(4-(4-biphenyl)butanoyl)-sn-glycero-3-phosphocholine (DBBPC), or tetradecanoyl-2-(4-(4-biphenyl)butanoyl)-sn-glycero-3-phosphocholine (TBBPC), in which one of the aliphatic chains contains a biphenyl unit. These phospholipids intrinsically have large positive $\Delta \chi$ inducing a spontaneous smectic orientation of bicelles in a magnetic field (part b of Figure 1). For these bicelle systems, detailed phase diagrams in terms of lipid ratio, temperature, total lipid concentration, and the effect of added salt were investigated by ${ }^{31} \mathrm{P} \mathrm{NMR}^{13,17,18}$ and their structural and orientation properties were studied by $\operatorname{NMR}\left({ }^{31} \mathrm{P},{ }^{14} \mathrm{~N}\right.$, and $\left.{ }^{2} \mathrm{H}\right)$, freeze fracture transmission electron microscopy (TEM), and SAXS. ${ }^{13}$ It was confirmed that the disklike bicelles were formed both with DMPC (about $40 \mathrm{~nm}$ in diameter), and with TBBPC (much larger - about $80 \mathrm{~nm}$ in diameter) as briefly described in the Materials and Method section below.

In this study, we follow by small-angle X-ray scattering the orientation kinetics in a magnetic field $(11.7 \mathrm{~T})$, as well as the relaxation kinetics of three types of bicelles, those formed with DMPC and DCPC: 1) with paramagnetic lanthanide $\mathrm{Eu}^{3+}$, (referred to as DMPC-Eu bicelles hereafter), and 2) without $\mathrm{Eu}^{3+}$ (referred to as DMPC bicelles), and 3) bicelles formed with TBBPC and DCPC (referred to as TBBPC bicelles). The results clearly indicate the complex interplay between morphology and ordering of the various bicelles. The larger the bicelles are, the more difficult it is to induce an ordering under a magnetic field. However, once the final ordering is attained, the time needed for bicelles to relax to isotropic orientation is surprisingly long and depends on the nature of the ordering rather than the size of the
Scheme 1. Molecular Structures of Three Lipids Used for Bicelle Preparation: DMPC, DCPC, and TBBPC

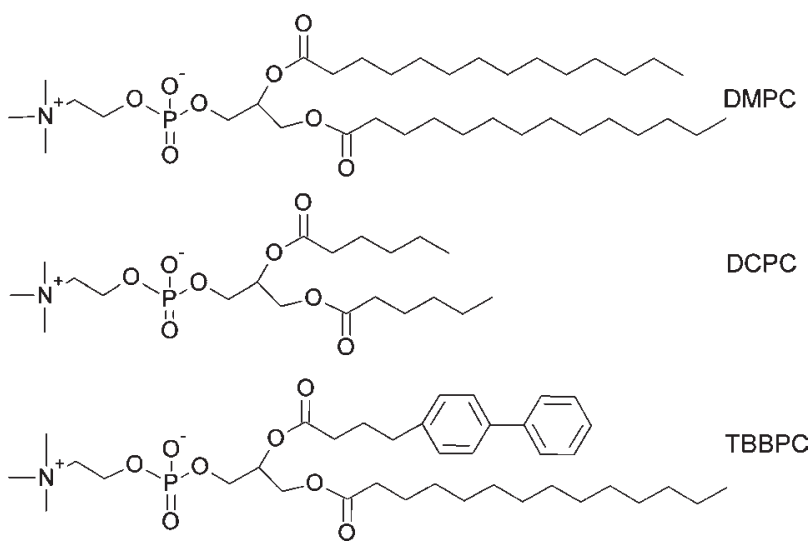

bicelles. Smectic ordering relaxes much more slowly than nematic ordering. Several days of remnant macroscopic orientation are observed outside the magnetic field, thus widely opening the field to structural biology and materials sciences. Detailed study of the SAXS profiles allows us to compare the different bicelle systems in terms of their morphologies at a molecular level. A theoretical account of this peculiar orientation behavior is also provided.

\section{MATERIALS AND METHODS}

Chemicals. 1,2-Dimyristoylphosphatidylcholine (DMPC) and 1,2-dicaproylphosphatidylcholine (DCPC) were purchased from Avanti Polar Lipids (Birmingham, AL, USA). These starting materials were used without further purification. The Europium chloride ions $\left(\mathrm{EuCl}_{3} 6 \mathrm{H}_{2} \mathrm{O}\right)$ were obtained from Aldrich Chemical Co. (Milwaukee, WI, USA). The phospholipid 1-tetradecanoyl2-(4-(4-biphenyl)butanoyl)-sn-glycero-3-phosphatidylcholine (TBBPC) was synthesized and purified following the procedure described by Loudet et al. (ref 13).

Bicelles Preparation. Appropriate amounts of phospholipids were weighted to obtain a mixture DMPC/DCPC with a mole content of DMPC of $78 \%$ (molar ratio $q=[\mathrm{DMPC}] /[\mathrm{DCPC}]=$ 3.5 ) and a mixture TBBPC/DCPC with a mole content of TBBPC of $88 \%(q=[\mathrm{TBBPC}] /[\mathrm{DCPC}]=7.3)$. A suitable volume of water, containing $100 \mathrm{mM} \mathrm{NaCl}$ or $\mathrm{KCl}$ and with the $\mathrm{pH}=6.5$ to mimic the physiological conditions was added to obtain a lipid hydration, $h$, of $80 \%$ for all preparations $(h=$ mass of water over the total mass of the system (phospholipids and water)). Hydrated samples were centrifuged at $6500 \mathrm{rpm}$ for $5 \mathrm{~min}$ and vigorously stirred in a vortex mixer. The samples were then frozen in liquid nitrogen for $30 \mathrm{~s}$, heated at $50^{\circ} \mathrm{C}$ for $10 \mathrm{~min}$ in a water bath, vigorously stirred in a vortex, and centrifuged again at $6500 \mathrm{rpm}$ for $5 \mathrm{~min}$. This cycle was repeated three times until a homogeneous suspension (viscous and translucent for DMPC/DCPC, fluid, and milky for TBBPC/DCPC) was obtained at room temperature. For the DMPC-Eu bicelles, a final concentration of $28 \mathrm{mM} \mathrm{Eu}^{3+}$ (molar ratio $\mathrm{DMPC} / \mathrm{Eu}^{3+}=1$ ) 0.09) was added to the DMPC/DCPC bicelles solution to flip them from the perpendicular to the parallel orientation relative to the magnetic field. Thirty microliters of each preparation was then transferred and sealed into a glass capillary with a $1.5 \mathrm{~mm}$ nominal diameter (Glaskapillaren GLAS, Germany) (Scheme 1). 
We have previously reported the phase diagrams both of DMPC/DCPC and TBBPC/DCPC mixture and have shown using NMR and freeze fracture TEM that at $35{ }^{\circ} \mathrm{C}$ at the hydration of $h=80 \%$, nanometric disklike structures (bicelles) existed for both systems. ${ }^{13,17}$

Estimation of the Size of Bicelles by ${ }^{31} \mathrm{P}$ NMR. The statistical size of bicelles, measured on TEM images, can be compared to that obtained from ${ }^{31} \mathrm{P}$ NMR data. Arnold et al. ${ }^{17}$ suggested a simple geometrical model, which allows determining bicelles diameter by directly integrating the area of the two sharp peaks, characteristic of bicelles on the ${ }^{31} \mathrm{P}$ NMR spectrum. This model considers that bicelles are perfectly aligned in the magnetic field and that there is a defined segregation between the two types of lipids: the long chains ones are located in the disk plane and are assigned to the highest peak of the spectrum and the short chains ones are diffusing in the disk torus and are assigned to the smallest peak. The molar ratio $q=$ [long chains lipids] $/[$ short chains lipids], used for the bicelles preparation, should be similar to the integration ratio between the two peaks on the ${ }^{31} \mathrm{P}$ NMR spectrum, also noted $q=$ [area of the disk plane $] /[$ area of the disk torus]. This ratio $q$ is linked to the bicelles diameter $\phi$ by the equation:

$$
\phi=\mathrm{aq}\left(\pi+\left(\pi^{2}+8 / \mathrm{q}\right)^{1 / 2}\right)+2 a
$$

where $a$ is the thickness of the lipid monolayer (for a phospholipid bilayers with a $\mathrm{C}_{14}$ aliphatic chain, $2 a \approx 40 \AA$.)

As it has been described in our previous papers, we have compared NMR diameters with the size of bicelles obtained by TEM observations, which showed good agreement both for DMPC/DCPC bicelles ${ }^{17}$ and TBBPC/DCPC bicelles. ${ }^{13}$

Orientation in the NMR Spectrometer and Transfer to the SAXS Apparatus. The bicelles orientation was induced by an 11.7 T magnetic field (Bruker Avance $500 \mathrm{MHz}$ spectrometer). The solid NMR probe, containing an empty $4 \mathrm{~mm}$ rotor, was regulated at $35{ }^{\circ} \mathrm{C}$ for $20 \mathrm{~min}$. A $1.5 \mathrm{~mm}$ glass capillary for X-ray scattering was filled with $30 \mu \mathrm{L}$ of bicelle preparation and was kept at $35^{\circ} \mathrm{C}$ for $20 \mathrm{~min}$ in a water bath. The capillary $\left(T=35^{\circ} \mathrm{C}\right)$ was then quickly transferred into the rotor within the NMR spectrometer, also regulated at $35^{\circ} \mathrm{C}$. The sample was then kept in the magnetic field at $35{ }^{\circ} \mathrm{C}$ for the desired time.

For the transfer from the NMR spectrometer to the SAXS apparatus, the capillary containing the bicelles was transferred from the rotor into the portable water bath regulated at $35^{\circ} \mathrm{C}$, then was mounted in the sample chamber of the SAXS apparatus, regulated at $35^{\circ} \mathrm{C}$. The time necessary for the transfer from NMR setup to SAXS apparatus was about $2 \mathrm{~min}$.

Small-Angle X-ray Scattering. A Rigaku Nanoviewer (Microsource generator, MicroMax 007, Tokyo, Japan), equipped with a rotating $\mathrm{Cu}$ anode generator operated at $40 \mathrm{kV}$ and $20 \mathrm{~mA}$, coupled with a Confocal Maxflux Mirror (selecting the $0.154 \mathrm{~nm}$ wavelength), and three sets of pinhole collimation was used. The beam size at sample position is fwhm ca. $200 \mu \mathrm{m}$. The $2 \mathrm{D}$ scattering pattern was measured using a Mercury CCD camera $(512 \times 512$ pixels, pixel size ca. $140 \mu \mathrm{m})$, and the sample-to-detector distance was fixed to $425 \mathrm{~mm}\left(0.02 \leq q \leq 0.35 \AA^{-1}\right)$. The exposure time was $600 \mathrm{~s}$ and the scattering intensities from the buffer solution, as well as the detector dark current, were measured separately and subtracted from those of the samples. The obtained 2D scattering patterns were analyzed with Rigaku Nanoviewer software, which allows an integration of the signal intensity to yield $I(q)$ graph (where $I$ is the scattering intensity and $q$ the scattering vector in reciprocal space). The resulting data were then treated with OriginLab software (Northampton, MA, USA).

\section{RESULTS}

Figure 1 shows schematically the isotropic orientation of bicelles before they are put in a magnetic field, then the two types of bicelles (nematic and smectic) with specific orientation induced in a magnetic field, which remained after their transfer in the SAXS apparatus. As it is clearly seen from the 2D scattering patterns obtained after transfer, the bicelle samples stay oriented outside the magnetic field long enough for the SAXS scattering patterns clearly showing the anisotropy. The $2 \mathrm{D}$ scattering patterns obtained for DMPC, DMPC-Eu, and TBBPC bicelles without magnetic orientation (A) all show a classical circular pattern, typical of non oriented bilayers. In the NMR spectrometer, the capillaries are put parallel to a $11.7 \mathrm{~T}$ magnetic field $\left(B_{0}\right)$ at $35^{\circ} \mathrm{C}$ (B), defined as $z$ axis. After their transfer in the SAXS apparatus, the capillaries containing bicelles, mounted perpendicular to the X-ray beam (beam axis along $y$ ) show scattering patterns as shown in Figure 1, with two strong vertical spots (in the direction of $z$ axis) for TBBPC bicelles and DMPC-Eu bicelles and two horizontal spots (in the direction of the $x$ axis) for DMPC bicelles. These scattering patterns indeed confirm the nematictype orientation of DMPC bicelles with the normal $\mathbf{n}$ of the lipid bilayer perpendicular to $B_{0}$, and smectic-type orientation of DMPCEu bicelles and TBBPC bicelles with their normals oriented parallel to the magnetic field.

It is noteworthy that scattered intensity of the anisotropic spots is much stronger for bicelles with smectic orientation than those having nematic orientation. This is because for SAXS, only the bicelles with the normal $\mathbf{n}$ of the lipid bilayer perpendicular to the X-ray beam participate in scattering, and, for the nematic orientation, this represents only a part of the bicelles population, whereas, for the smectic orientation, all of the bicelles participate to the scattering.

Kinetics of Orientation of Bicelles in a Magnetic Field. We followed the kinetics of bicelle orientation in the magnetic field. In Figure 2, scattering patterns of bicelles are recorded after 5, 10, 15 , and $40 \mathrm{~min}$ in the $11.7 \mathrm{~T}$ magnetic field. Using the variation of the scattered intensity radially integrated between $q=0.07 \AA^{-1}$ and $q=0.28 \AA^{-1}$ and plotted as a function of angle $\theta$ with respect to the vertical axis (top of the screen is defined as angle $0 \mathrm{deg}$ ), the anisotropy of the orientation was quantified as follows:

$$
\operatorname{anisotropy}(t)=\frac{I_{\max }-I_{\min }}{I_{\max }+I_{\min }}
$$

with $I_{\max }$ and $I_{\min }$ the maximum and minimum (time-dependent) values respectively of the intensity found in azimuthal plots. Interestingly, both DMPC and DMPC-Eu bicelles reached $>70 \%$ of their final orientation in less than $5 \mathrm{~min}$, whereas the TBBPC bicelles were slower to reach theirs $(\sim 15 \mathrm{~min})$. However, Figure 2 indicates that both bicelles exhibiting a smectic orientation (DMPC-Eu and TBBPC) have a much higher anisotropy, typically $\geq 0.8$, than DMPC bicelles showing a nematic orientation and an anisotropy $\sim 0.35$.

Kinetics of Relaxation of Bicelles Orientation Outside the Magnetic Field. The relaxation kinetics of oriented bicelles was investigated once the maximum anisotropy-orientation was reached under the magnetic field. All of the bicelles have been subjected to an $11.7 \mathrm{~T}$ magnetic field for $15 \mathrm{~min}$; the relaxation was then followed with SAXS as shown in Figure 3. 

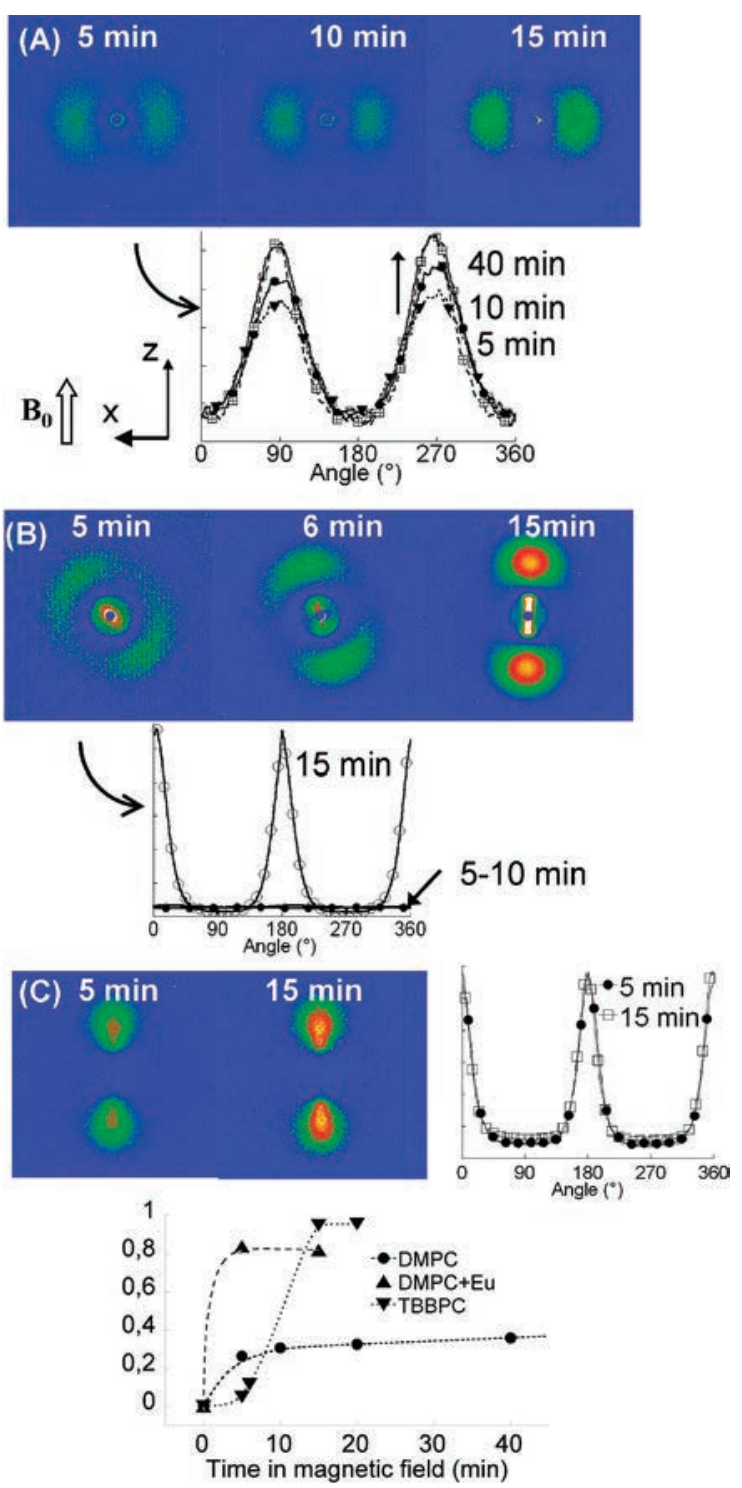

Figure 2. SAXS pattern of (A) DMPC bicelles, (B) TBBPC bicelles, and (C) DMPC-Eu bicelles, which were subjected to an $11.7 \mathrm{~T}$ magnetic field $\left(B_{0}\right)$ during different durations. The graphs show the variation of the integrated scattered intensity as a function of the azimuthal angle $\theta$. The graph at the bottom shows the kinetics of anisotropy growth.

The DMPC bicelles relaxed to an isotropic orientation in about one hour where no more detectable anisotropy was observed. On the contrary, the TBBPC bicelles kept their anisotropy even after several days. Indeed, the isotropic orientation was observed only after 10 days! Interestingly, a similar very slow kinetics was observed with DMPC-Eu bicelles although only the first four hours could be followed.

To better quantify the relaxation kinetics, the evolution of anisotropy as a function of time is presented for the different systems in Figure 4. To facilitate the comparison, the anisotropy is normalized with its initial value (the highest value of the anisotropy) before relaxation. For DMPC bicelles, the relaxation time depended on the time the bicelles were oriented in the magnetic field, that is, the longer the bicelles were oriented in the magnetic field, the slower the relaxation was. Beyond $40 \mathrm{~min}$, however, the relaxation time did not vary any more.

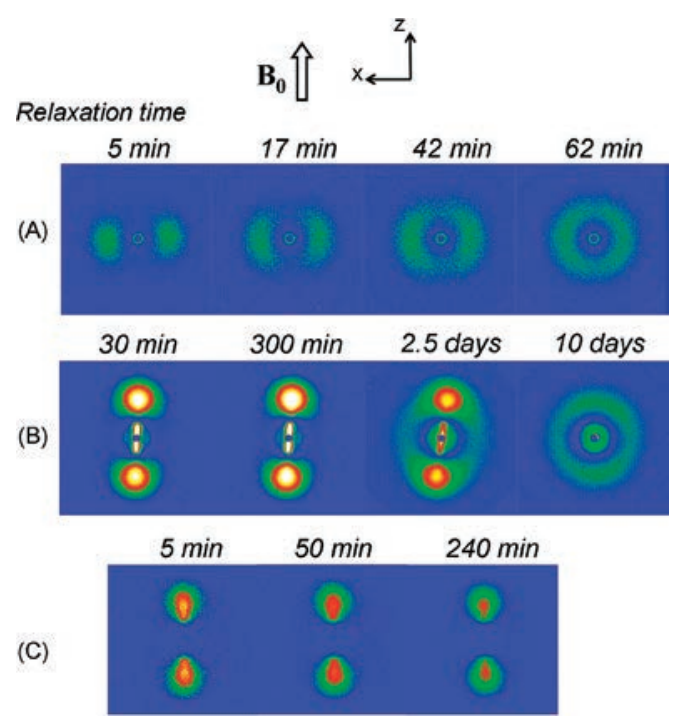

Figure 3. SAXS scattering patterns of (A) DMPC bicelles, (B) TBBPC bicelles, and (C) DMPC-Eu bicelles during their relaxation outside $B_{0}$ after $15 \mathrm{~min}$ of orientation in an $11.7 \mathrm{~T}$ magnetic field at $35^{\circ} \mathrm{C}$.
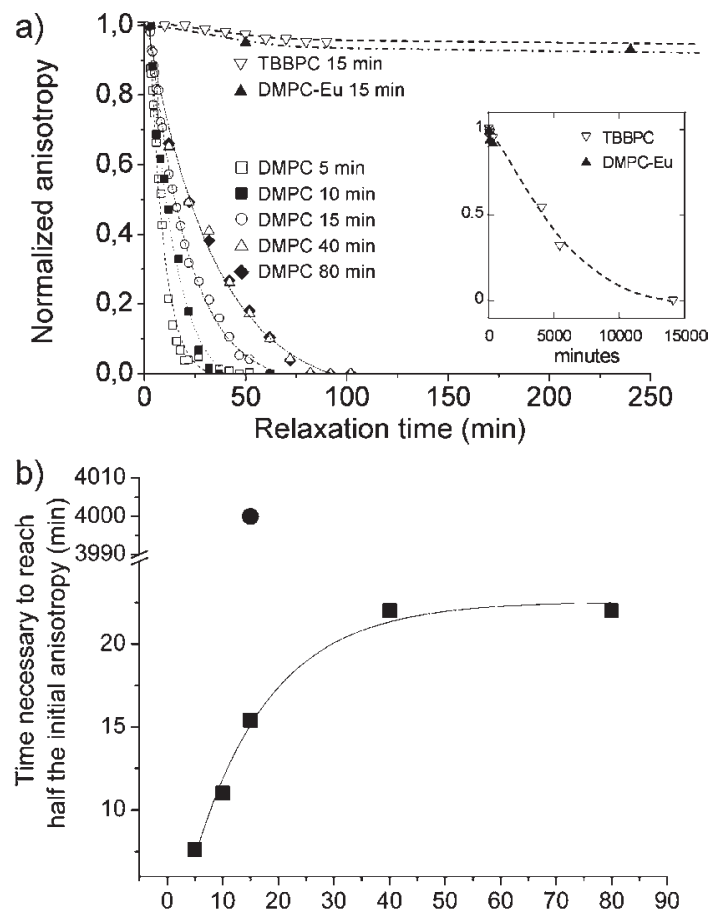

orientation time of bicelles in 11,7T magnetic field (min)

Figure 4. a) Normalized anisotropy as function of the time ( $\mathrm{min})$ for DMPC bicelles after $5 \mathrm{~min}(\square), 10 \mathrm{~min}(\boldsymbol{\square}), 15 \mathrm{~min}(\bigcirc), 40 \mathrm{~min}(\Delta)$, and $80 \mathrm{~min}(\checkmark)$ of orientation in an $11.7 \mathrm{~T}$ magnetic field $B_{0}$ and for TBBPC bicelles $(\nabla)$ and DMPC-Eu bicelles $(\boldsymbol{\Delta})$ after $15 \mathrm{~min}$ of orientation in an $11.7 \mathrm{~T}$ magnetic field $B_{0}$. b) Time necessary for the DMPC bicelles ( $\mathbf{\square})$ and TBBPC bicelles (O) to relax to half the initial anisotropy as a function of the orientation time in the magnetic field.

Analysis of Scattering Patterns. The 2D scattering patterns have been azimuthally integrated to yield $I(q)$ graphs. Integration is performed over $0-360^{\circ}$ for nonoriented systems and over $80-100^{\circ}$ with the direction perpendicular to the magnetic field 
( $x$ axis) for DMPC bicelles with nematic orientation or $170-190^{\circ}$ along the direction parallel to $B_{0}(z$ axis) for TBBPC and DMPCEu bicelles with smectic orientation.

The different experimental $I=f(q)$ patterns (circles) of nonoriented and oriented DMPC, TBBPC, and DMPC-Eu bicelles are shown in Figure 5. For all of the systems, a broad peak between $q$ ca. 0.05 and $q=0.35 \AA^{-1}$ is observed, which is characteristic of a phospholipid bilayer structure. ${ }^{19-21}$

For the TBBPC bicelles, a Bragg peak is clearly observed at $q=$ $0.055 \AA^{-1}$, which corresponds to a $1 \mathrm{D}$ stacking periodicity of $114 \AA$ A. This Bragg peak is much less pronounced for the nonoriented TBBPC bicelles. For the DMPC-Eu bicelles, a weak shoulder could be observed at around $0.1 \AA^{-1}$.

There are several distinct models for fitting the SAXS scattering patterns obtained with periodically stacked lipid bilayers. $^{22,20,23-25}$ Whereas the models based on smooth electronic density profiles presumably give a more realistic vision of the membrane structure than the so-called strip models introduced by Worthington, ${ }^{26}$ it has early been noticed that "the results of the analysis are not sensitive to guessing precisely the unknown functional form for the electron density of the bilayer" - as quoted from Wiener et al. ${ }^{27}$ Because the scattering patterns are limited to a relatively narrow scattering vector range and, as is obvious in Figure 5, do not exhibit more than, at best, one Bragg line, we have chosen to use a simple strip model with, in addition, a physical description of the stacking disorder as introduced in ref 22. Such a choice combines a number of adjustable parameters commensurate with the scattering data quality to the requirement of using Caille theory in modeling the spectra. ${ }^{28,25,29}$

To fit the scattering patterns, we have thus used the following, three-parameter form factor: $P(q)=\left[\sin \left(q^{*} \delta_{\mathrm{T}}\right) \sin \left(q\left[\delta_{\mathrm{T}}+\right.\right.\right.$ $\left.\left.\left.\delta_{\mathrm{H}}\right]\right)-\sin \left(q \delta_{\mathrm{T}}\right) \sin \left(q^{*}\left[\delta_{\mathrm{T}}+\delta_{\mathrm{H}}\right]\right)\right] / q^{2}$ where $\delta_{\mathrm{H}}$ and $\delta_{\mathrm{T}}$ are defined in the strip model as the thickness of polar head groups and hydrophobic tails, respectively. The parameter $\mathrm{q}^{*}$ corresponds mathematically to the location in reciprocal space of the form factor first minimum and its value is straightforwardly read with good accuracy from the experimental data. It is also physically associated to the electron density contrast ratio $\delta \rho_{\text {tail }} / \delta \rho_{\text {head }}=\left(\rho_{\text {tail }}-\rho_{\text {water }}\right) /\left(\rho_{\text {head }}-\rho_{\text {water }}\right)$ by the relation $\left(\sin \left(q^{*}\left[\delta_{\mathrm{T}}+\delta_{\mathrm{H}}\right]\right)\right) /\left(\sin \left(q^{*} \delta_{\mathrm{T}}\right)=1-\left(\delta \rho_{\text {tail }}\right) /\left(\delta \rho_{\text {head }}\right)\right.$. In the cases where Bragg peaks are visible, a structure factor term $S(q)$, namely eq (17) of ref 22 for a finite-size bilayer stack with elastic distortions, is used. This increases the number of physical parameters by three, with the stack periodicity, the size of the lamellar stack along the stacking axis and the Caillé exponent, describing the strength of thermal disorder and - ultimately the simultaneous broadening and damping of the Bragg peaks, coming into play. The complete model, including two instrumental parameters (a scaling factor $\mathrm{k}$ and a background intensity $\left.I_{\mathrm{b}}\right)$ is therefore of the following form $I(q)=k P(q) S(q) / q^{2}+I_{\mathrm{b}}$ with a total of 8 independent parameters. Note that this model treats bicelles as infinite objects with a broad orientational distribution, as testified by the $1 / q^{2}$ Lorentz factor normalization accounting for powder averaging. Therefore, it does not give any information on the (lateral) size distributions of the bicelles. ${ }^{30}$ This approach can be justified by the bicelles size which ranges between 40 to $80 \mathrm{~nm}$, too large to enter (from below) the $q$ range, which was used for the fit.

The results of the fits to the scattering patterns are shown in Figure 5 as solid lines and values for the resulting three contrast parameters summarized in Table 1 . With many freely adjustable parameters in the model and a restricted $q$ range, several distinct
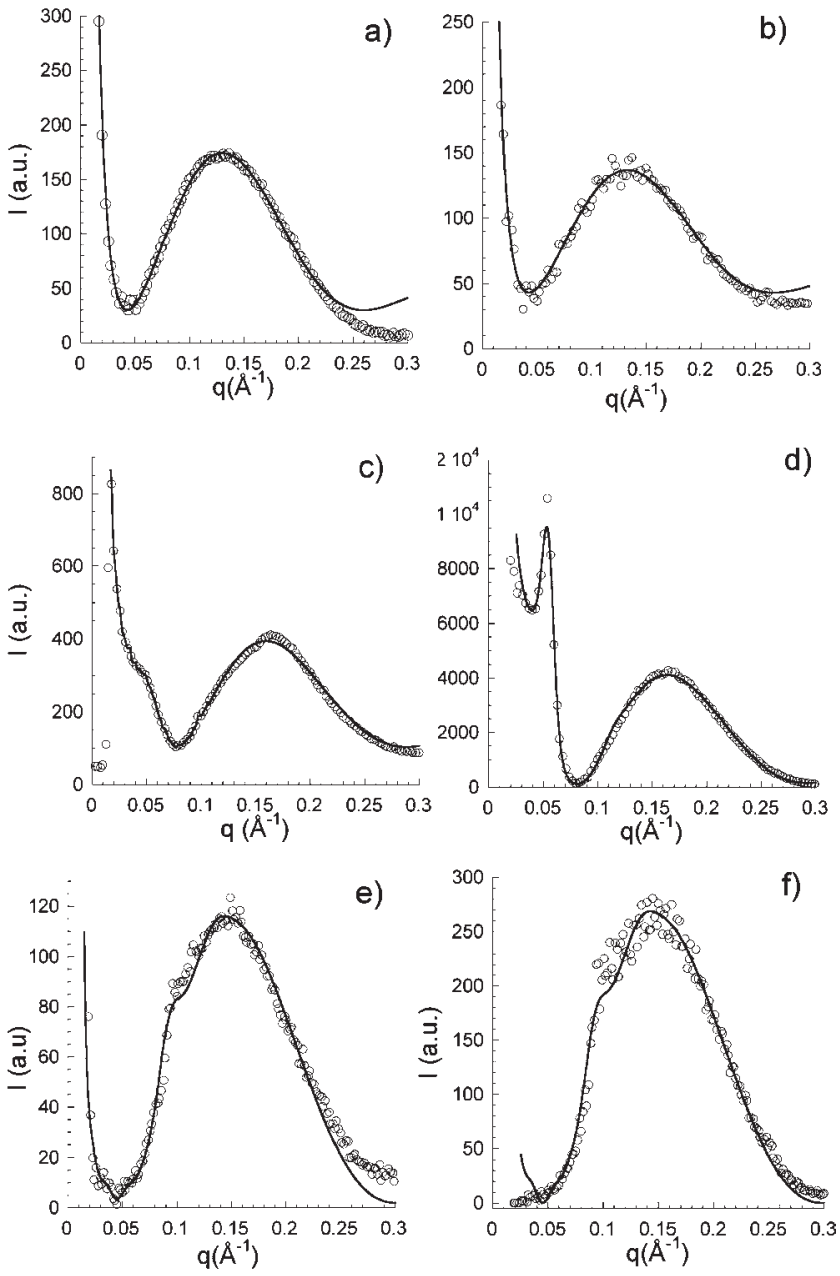

Figure 5. $I=f(q)$ experimental profiles (circles) and simulated profiles (solid line) of a) DMPC bicelles without orientation (circular integration), b) DMPC bicelles after $80 \mathrm{~min}$ in $B_{0}$ and an angular integration between 80 and $100^{\circ}$ along the $x$ axis, c) TBBPC bicelles without orientation (circular integration), d) TBBPC bicelles after 20 min in $B_{0}$ and an angular integration between 170 and $190^{\circ}$ along the $z$ axis. e) DMPC-Eu bicelles without orientation (circular integration), f) DMPCEu bicelles after $15 \mathrm{~min}$ in $B_{0}$ and an angular integration between 170 and $190^{\circ}$ along the $z$ axis.

fitting solutions are possible. A simplifying hypothesis was thus made to reduce the number of unconstrained parameters. Because both DMPC and TBBPC have phosphocholine head groups in common, we fixed the headgroup thickness $\delta_{\mathrm{H}}$ to be $5 \AA$ for all systems, ${ }^{31}$ reducing to 7 the actual number of fitted parameters. As a matter of fact, no fit is actually required for parameter $q^{*}$ because its value obtains directly from the data, and 3 structure factor parameters are irrelevant with DMPC bicelles. The number of a priori unconstrained parameters therefore differs for the three systems, respectively 3 for DMPC, 5 for TBBPC (the stacking period is read from the data), and 6 for DMPC-Eu. In all three cases, we estimate uncertainties associated to the quoted $\delta_{\mathrm{T}}$ values to be $\pm 0.1 \AA$, and $\pm 0.002 \AA^{-3}$ for $\rho_{\text {head }}$.

For the two kinds of DMPC bicelles, the scattering pattern analysis resulted in a hydrophobic thickness to be $\delta_{\mathrm{T}} 15.1$ and 14.7 Å for isotropic and oriented bicelles respectively leading to a bilayer thickness of about $40 \AA$. These value are in accordance with early measurements made on neutron diffraction profiles of 
Table 1. Parameters Used for the $I=f(q)$ Simulated Profiles of Nonoriented and Oriented DMPC, TBBPC, and DMPC-Eu Bicelles $^{a}$

\begin{tabular}{|c|c|c|c|c|c|c|c|c|}
\hline & $\alpha=\delta \rho_{\text {tail }} / \delta \rho_{\text {head }}$ & $\delta_{\mathrm{H}}(\AA)$ & $\delta_{\mathrm{T}}(\AA)$ & $\rho_{\text {tail }}$ & $\rho_{\text {head }}$ & $\rho_{\mathrm{H} 2 \mathrm{O}}$ & bilayer thickness $(\AA ̊)$ & periodicity $(\AA)$ \\
\hline DMPC & -0.258 & 5.0 & 15.1 & 0.260 & 0.601 & 0.33 & 40 & \\
\hline DMPC oriented & -0.274 & 5.0 & 14.7 & 0.260 & 0.585 & 0.33 & 39 & \\
\hline ТВВРC & -0.274 & 5.0 & 13.6 & 0.270 & 0.549 & 0.33 & 37 & 114 \\
\hline TBBPC oriented & -0.274 & 5.0 & 13.4 & 0.270 & 0.549 & 0.33 & 37 & 114 \\
\hline DMPC-Eu & -0.395 & 5.0 & 12.8 & 0.260 & 0.592 & $0.354(+\mathrm{Eu})$ & 36 & 135 \\
\hline DMPC-Eu oriented & -0.391 & 5.0 & 12.8 & 0.260 & 0.594 & $0.354(+\mathrm{Eu})$ & 36 & 135 \\
\hline
\end{tabular}

${ }^{a}$ Slanted numbers correspond to values kept fixed during the fitting procedure. $\delta_{\mathrm{H}}$ and $\delta_{\mathrm{T}}$ are the thickness of head groups and tails respectively, $\alpha$ is the ratio of differences between the electronic density of tails and head groups with water, $\delta \rho_{\text {tail }} / \delta \rho_{\text {head }}=\left(\rho_{\text {tail }}-\rho_{\text {water }}\right) /\left(\rho_{\text {head }}-\rho_{\text {water }}\right) \cdot \rho_{\text {tail }}, \rho_{\text {head }} \rho_{\text {water }}$ are electronic densities of lipid tail, head group, and water, respectively.

DMPC bilayers ${ }^{31,32}$ With the value for $q^{*}$ quite well-defined in the experimental spectra, the contrast ratio is then easily obtained. To get absolute values for the electron densities, either $\rho_{\text {tail }}$ or $\rho_{\text {head }}$ has to be fixed. In the case of DMPC at $35^{\circ} \mathrm{C}$, we can consider $\rho_{\text {tail }}$ to be close to the electron density for alkyl chains, $\sim 0.26 \AA^{-3}$, which gives $\rho_{\text {head }} \sim 0.60 \AA^{-3}$ (isotropic) and $0.59 \AA^{-3}$ (oriented).

In the case of TBBPC bicelles, the obtained thicknesses of the hydrophobic chain domains were 13.6 and $13.4 \AA$ for the isotropic and oriented states, respectively. The hydrophobic core electron density $\rho_{\text {tail }}$ of the TBBPC bilayers is chosen from the following argument: as TBBPC has one wholly alkyl chain, the other one containing a biphenyl group, we used a value $0.27 \AA^{-3}$, intermediate between the electron density for benzene $(0.285)$ and for alkyl (0.26) chains. This choice led to $\rho_{\text {head }}=0.55 \AA^{-3}$.

The magnetically oriented TBBPC bicelles exhibit a marked Bragg peak at around $0.05 \AA^{-1}$, implying the relevance of the structure factor term in the model. With a periodicity fixed to $d=114 \AA$, a very good fit was obtained. Meanwhile, for the DMPC-Eu bicelles, the shoulder observed at around $0.1 \AA^{-1}$ turned out to be the second-order Bragg peak. It was not possible to satisfactorily fit the scattering pattern with the shoulder at 0.1 as the first-order Bragg peak. Interestingly, the minimum of the scattering pattern at around $0.050 \AA^{-1}$ due to the form factor canceled the first-order peak which was expected, from the (fitted) periodicity of $135 \AA$, at $0.047 \AA^{-1}$. For the calculation of the electron density of these bicelles, some care has to be taken. The presence of added Europium ions, which likely condense at the surface of the bicelles, would probably increase the electron density of water, $\rho_{\text {water }}$. We therefore looked for a $\rho_{\text {water }}(+\mathrm{Eu})$ value, which would give a $\rho_{\text {head }}$ value similar to what was obtained with DMPC bicelles using $\rho_{\text {tail }}=0.26 \AA^{-3}$ and $\delta \rho_{\text {tail }} / \delta \rho_{\text {head }}$ obtained from the fit. This consistently led to $\rho_{\text {water }}(+\mathrm{Eu})=0.354 \AA^{-3}$, indeed slightly higher than the electron density of water to $\rho_{\text {water }}=0.33 \AA^{-3}$.

\section{DISCUSSION}

The three bicelle systems, DMPC, TBBPC, and DMPC-Eu, showed interesting differences in terms of organization and kinetics of orientation and relaxation. Both TBBPC bicelles and DMPC-Eu bicelles showed smectic orientation (and a smectic local structure) compared to DMPC bicelles with nematic orientation. In terms of obtained anisotropy, as defined above, clearly a difference was observed between the two orientations, whereas the DMPC bicelles reached an anisotropy of about 0.35 , both bicelle types with smectic organization reached much higher anisotropies, 0.8 for DMPC-Eu and 0.95 for TBBPC. Such an important variation in anisotropy may tentatively be ascribed to the intrinsic differences between the nematic ordering of discs, where a $2 \mathrm{D}$ degeneracy is kept for the membrane normal - a small fraction of discs actually participate to the signal increase and smectic ordering where all of the membranes are organized in essentially the same direction. The smaller but detectable difference in anisotropy between DMPC-Eu and TBBPC can be attributed to the smaller size of DMPC-Eu bicelles (40 nm vs $80 \mathrm{~nm}$ ) which may have higher disorder due to thermal fluctuations. These qualitative arguments are quantitatively supported by a simple model by introducing the appropriate orientation distribution functions for the normal $\mathbf{n}$ to the lipid bilayers, namely $f_{\text {nem }}(\mathbf{n})$ with a peak when $\mathbf{n}$ lies in the $x y$ plane for the nematic orientation, and $f_{\text {smect }}(\mathbf{n})$ with a peak when $\mathbf{n}$ is along (or opposite to) the $z$ axis (smectic orientation). The intensity recorded at a given detector pixel, corresponding to a scattering wave vector $\mathbf{q}$, is indeed given by: $I(\mathbf{q}) \propto \int d^{2} \mathbf{n} f(\mathbf{n}) I_{\mathbf{n}}(\mathbf{q})$, where $I_{\mathbf{n}}(\mathbf{q})$ represents the intensity scattered at the same detector pixel by a single bicelle oriented along $\mathbf{n}$. For a bicelle with a finite lateral extension, $I_{\mathbf{n}}(\mathbf{q})$ is given by the product $P\left(q_{z}\right) \times P_{\perp}\left(\mathbf{q}_{\perp}\right)$ of the (usual) form factor across the bilayer (i.e., parallel to the normal $\mathbf{n}$ ), namely $P\left(q_{z}\right)$, by the transversal form factor $P_{\perp}\left(\mathbf{q}_{\perp}\right)$ accounting for the finite (though large) in-plane dimensions of the lipid platelet. Geometrically, the wave vector (or wave vector component) are expressed as $q_{z}=\mathbf{q} \cdot \mathbf{n}$ and $\mathbf{q}_{\perp}=\mathbf{q}-q_{z} \mathbf{n}$, whereas, for large enough platelets, the transversal form factor is strongly peaked at $\mathbf{q}_{\perp}=\mathbf{0}$. For instance, modeling the bicelles as homogeneous, flat cylinders of radius $R$, the transversal form factor is explicitly obtained in terms of the Bessel function of integer order 1 as $P_{\perp}\left(q_{\perp}\right)=4\left(J_{1}^{2}\left(q_{\perp} R\right)\right) /\left(q_{\perp} R\right)^{2}$ and the intensity recorded at a given detector pixel becomes $I(\mathbf{q}) \propto \int \mathrm{d}^{2}$ n $f(\mathbf{n}) P(\mathbf{q} \cdot \mathbf{n}) 4\left(J_{1}{ }^{2}\left[\left(q^{2}-[\mathbf{q} \cdot \mathbf{n}]^{2}\right)^{1 / 2} R\right]\right) /\left(\left(q^{2}-[\mathbf{q} \cdot \mathbf{n}]^{2}\right) R^{2}\right)$. In the approximation where the transversal form factor is considered as equivalent to a Dirac delta function, which is asymptotically valid when $q R \gg 1$, the expression writes:

$$
\begin{aligned}
I(\mathbf{q}) & \propto[f(\mathbf{q} / q)+f(-\mathbf{q} / q)] P(q) \int \mathrm{d}^{2} \mathbf{n} 4 \frac{J_{1}^{2}\left[\left(q^{2}-[\mathbf{q} \cdot \mathbf{n}]^{2}\right)^{1 / 2} R\right]}{\left(q^{2}-[\mathbf{q} \cdot \mathbf{n}]^{2}\right) R^{2}} \\
& \approx \frac{8 \pi}{q^{2} R^{2}}[f(\mathbf{q} / q)+f(-\mathbf{q} / q)] P(q)
\end{aligned}
$$

using the integral identity $\int_{0}^{\infty}\left[\left(J_{1}(x)\right) /(x)\right]^{2} x \mathrm{~d} x=1 / 2$. The classical $1 / q^{2}$ Lorentz factor appropriate for powder averaging is thus recovered for an isotropic orientation distribution 

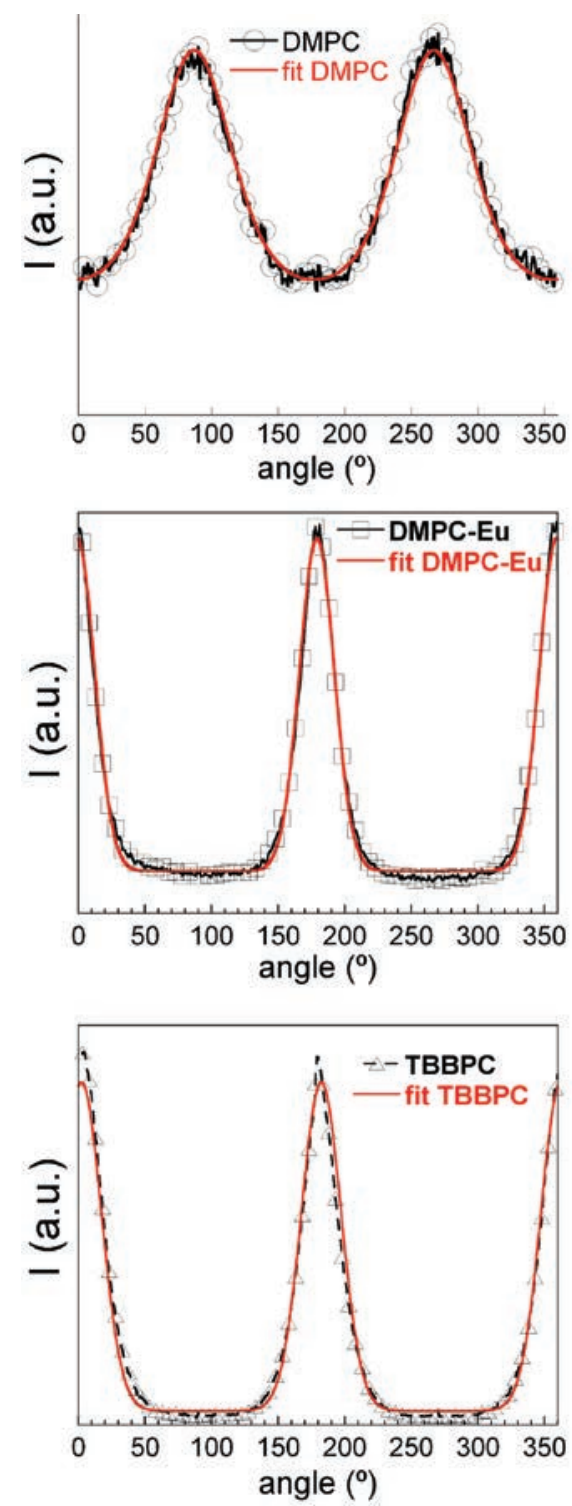

Figure 6. Radially averaged spectra in the long-time limit and fits to the nematic or smectic orientational distribution functions, as appropriate, for the DMPC, DMPC-Eu, and TBBPC bicelles.

function where $f(\mathbf{n})=1 / 4 \pi$. In the case of oriented bicelles, the anisotropy parameter as defined in the Results section is predicted to be $(|f(\hat{z})-f(\hat{x})|) /(f(\hat{z})+f(\hat{x}))$ with $\hat{z}$ (respectively $\hat{x})$ the unit vector along the vertical (respectively horizontal) detector axis. To be explicit and proceed further, we introduce as a plausible model for $f_{\text {smect }}(\mathbf{n})$ a function derived from the Onsager orientation distribution function, namely

$$
f_{\text {smect }}(\theta, \phi)=\frac{1}{4 \pi}\left(1+C\left[\frac{\alpha \cosh (\alpha \cos \theta)}{\sinh \alpha}-1\right]\right)
$$

and, similarly,

$$
f_{\text {nem }}(\theta, \phi)=\frac{1}{4 \pi}\left(1+C\left[\sqrt{\frac{2 \alpha}{\pi}} e^{-\alpha \frac{\cos ^{2} \theta}{2}}-1\right]\right)
$$

In both cases, $\alpha$ characterizes the width of the (nearly Gaussian) angular distribution with respect to the polar axis (smectic case) or
Table 2. Values for $C$ and $\alpha$ Resulting from the RadiallyIntegrated Scattering Intensities Obtained from the Data Shown in Figure 2

\begin{tabular}{lrc} 
& $\alpha$ & $C$ \\
DMPC & 4.3 & 0.60 \\
TBBPC & 15.0 & 0.62 \\
DMPC-Eu & 19.4 & 0.29 \\
\hline
\end{tabular}

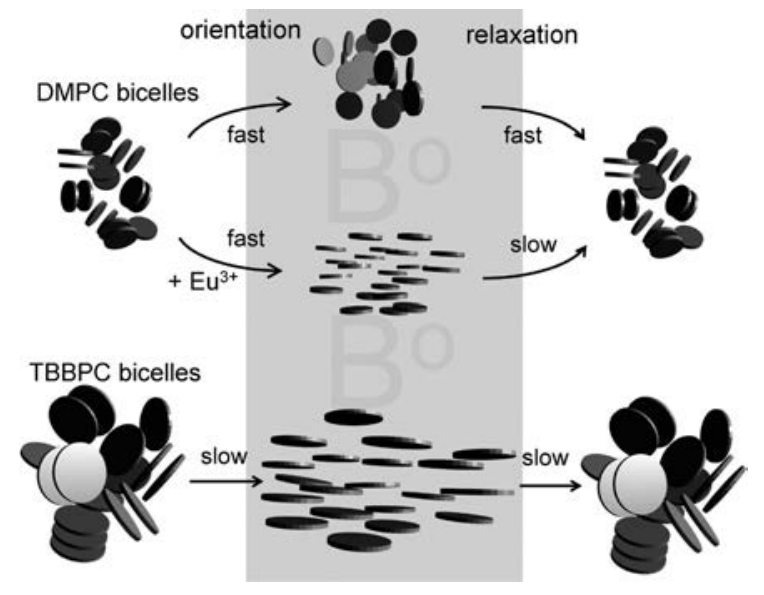

Figure 7. Schematic representation of kinetics of orientation and relaxation as a function of bicelles size and ordering.

equatorial plane (nematic case), and $C$ (a positive number less than 1) is associated to the relative weight of the oriented population; the higher $\alpha$, the higher the bicelle macroscopic ordering. The radially averaged data as displayed in Figure 2, that is over an extended $q$-range significantly larger than the close vicinity of the first-order Bragg peak, if any, has been fitted in the long-time limit using $f_{\text {smect }}(\mathbf{n})$ or $f_{\text {nem }}(\mathbf{n})$ as appropriate. The resulting graphs are displayed in Figure 6 , and the obtained values for $\mathrm{C}$ and $\alpha$ are summarized in Table 2 .

A much narrower angular spread for the two smectic states (TBBPC and DMPC+Eu bicelles) is observed as compared to the nematic one (DMPC bicelles), as evidenced by much larger $\alpha$ values. The data on the relaxation kinetics of the anisotropy as discussed previously nicely complements this result. The smectic orientation shows a much slower relaxation (days), whereas the DMPC bicelles with nematic orientation show fast relaxation (hour). Clearly, the higher smectic ordering required longer relaxation times.

Interestingly, for the kinetics of the bicelles orientation under a magnetic field, other factors seem to come into play, since in this case, both DMPC bicelles and DMPC-Eu bicelles had more rapid kinetics $(\sim 5 \mathrm{~min})$ compared to TBBPC bicelles (15 min). To understand this difference, it is useful to compare the bicelle sizes as both kinds of DMPC bicelles showing rapid orientation kinetics are indeed smaller than the TBBPC bicelles. Probably steric effects play an important role for slowing down the initial stage of orientation. A schematic representation of the relation between bicelle sizes and kinetics of orientation and relaxation is presented in Figure 7.

For either the DMPC or the TBBPC systems, a moderate proportion (1-C $\sim 40 \%$ ) of bicelles remains unaffected by the magnetic field. The proportion of oriented bicelles is twice less for 
the DMPC-Eu system, reaching only ca. 30\%: this explains why its anisotropy parameter, a combination of $C$ and $\alpha$ with a value about $80 \%$ in the present case, is less than for the TBBPC system (anisotropy parameter above 90\%) in spite of a narrower angular spread.

The scattering pattern analysis gave some other interesting information on the morphologies of bicelles. For all the bicelles, the bilayer thicknesses estimated from the form factor were between 35 to $40 \AA$. The values obtained for DMPC bicelles $(\sim 40 \AA)$ are in good agreement with what was expected from the literature $(43.4 \AA) .{ }^{10}$ The bilayers of TBBPC seem to be a little thinner than those of DMPC, which is expected from the shorter biphenyl chains which would lead to statistically slightly thinner hydrophobic core of the bilayers. For both TBBPC bicelles and DMPC-Eu bicelles, along with the bilayer form factor, a peak originating from the structure factor representative of the periodic ordering of bilayers was observable not only for the bicelles with smectic orientation but also for isotropic samples. This indicated that even for the bicelles with isotropic organization, locally shortrange stacking (with ca. 3 to 5 stacked bilayers for isotropic organization, and up to about 20 for the smectic ordering of TBBPC bicelles) existed. As the diameters of the bicelles are $40 \mathrm{~nm}$ for DMPC bicelles and $80 \mathrm{~nm}$ for TBBPC bicelles, whereas the observed periodicity was of the order of a hundred angstroms at the studied concentration, and these solutions are much beyond the overlap concentration, which explains the presence of local ordering. This may indeed explain the fact that all these bicellar samples are also optically birefringent and have translucent aspects, they probably have a local ordering, which spans about a thousand angstroms (several stacked bilayers). Here, we have to point out that such local organization can lead to periodicities of Bragg peaks, which are smaller than what is expected from lamellar phases with homogeneous distribution of lipids. ( $\sim 180 \AA$ instead of 114 or $135 \AA$ as observed). The electronic densities of head groups and hydrophobic tails were also obtained. For both of the DMPC bicelles, the calculated electronic densities of head groups $\rho_{\mathrm{H}}$ are around $0.59-0.6 \AA^{-3}$ for a fixed value of $\rho_{\mathrm{T}}=0.26 \AA^{-3}$, whereas the $\rho_{\mathrm{H}}$ of TBBPC was slightly smaller $\sim 0.55 \AA^{-3}$ for a fixed value of $\rho_{\mathrm{T}}=0.27 \AA^{-3}$ (a value estimated from mixed hydrophobic chains including aromatic and aliphatic groups). The slight decrease in the $\rho_{\mathrm{H}}$ value may be due to a bulkier shape of TBBPC, which separates the head groups farther apart.

\section{CONCLUSIONS}

We have reported the structural study of nanometric bilayer discs bicelles formed with different phospholipids systems (DMPC, DMPC-Eu, TBBPC bicelles) and the study on the kinetics of ordering of bicelles induced by magnetic field and their relaxation once they are outside the magnetic field. The SAXS analysis clearly revealed the local structure of lipid bilayers for all the three bicelles systems. Compared to the DMPC bicelles, the TBBPC bicelles have slightly thinner hydrophobic core with higher electron density due to the presence of biphenyl groups. The condensation of $\mathrm{Eu}^{3+}$ ions at the membrane surface of DMPC-Eu was also clearly observed.

The SAXS measurements clearly demonstrated the nematic orientation of DMPC bicelles in a magnetic field, whereas TBBPC bicelles and DMPC-Eu bicelles showed smectic orientation. The investigation on their orientation/relaxation kinetics indicated that the orientation mechanism seems to be governed more by the size of the bicelles, as the smaller bicelles, DMPC bicelles, and DMPC-Eu bicelles, have similar and fast orientation kinetics, (about $5 \mathrm{~min}$ ) compared to the larger bicelles, TBBPC bicelles (about $15 \mathrm{~min}$ ). The steric hindrance between disks may be playing a major role for decelerating the formation of the longrange ordering from the isotropic organization under magnetic field and this effect is more important for the larger bicelles. However, the relaxation mechanism as well as the orientational anisotropy did not follow the same rule. The relaxation of bicelles with smectic orientation DMPC-Eu bicelles and TBBPC bicelles was very slow and the anisotropic orientation can be detected even after a few days outside the magnetic field in particular for the TBBPC bicelles, whereas the DMPC bicelles with nematic orientation relaxed back to isotropic orientation after about one hour. Such a remnant long-lived orientation of bicelles outside the magnetic was reported for the first time here and the striking difference in the relaxation property between different bicelles systems can be due to the higher degree of organization of the smectic arrangement with respect to nematic one as it was demonstrated by the analysis of anisotropic scattering pattern. Indeed, the detailed investigation of scattered intensity clearly indicated that much higher ordering is observed with the bicelles with smectic orientation with respect to nematically oriended bicelles.

\section{AUTHOR INFORMATION}

\section{Corresponding Author}

*E-mail: r.oda@iecb.u-bordeaux.fr.

\section{ACKNOWLEDGMENT}

We gratefully thank Brice Kauffmann (IECB, Pessac), for his help in SAXS experiments.

\section{REFERENCES}

(1) Dubois, M.; Lizunov, V.; Meister, A.; Gulik-Krzywicki, T.; Verbavatz, J. M.; Perez, E.; Zimmerberg, J.; Zemb, T. Proc. Natl. Acad. Sci. U.S.A. 2004, 101, 15082.

(2) Gabriel, N. E.; Roberts, M. F. Biochemistry 1984, 23, 4011.

(3) Sanders, C. R.; Prosser, R. S. Structure 1998, 6, 1227.

(4) Sanders, C. R.; Landis, G. C. Biochemistry 1995, 34, 4030.

(5) Opella, S. J.; Zeri, A. C.; Park, S. H. Annu. Rev. Phys. Chem. 2008, 59, 635.

(6) Park, S. H.; De Angelis, A. A.; Nevzorov, A. A.; Wu, C. H.; Opella, S. J. Biophys. J. 2006, 91, 3032.

(7) Mahalakshmi, R.; Marassi, F. M. Biochemistry 2008, 47, 6531.

(8) Cardon, T. B.; Tiburu, E. K.; Padmanabhan, A.; Howard, K. P.; Lorigan, G. A. J. Am. Chem. Soc. 2001, 123, 2913.

(9) Hare, B. J.; Prestegard, J. H.; Engelman, D. M. Biophys. J. 1995, 69, 1891.

(10) Kucerka, N.; Liu, Y. F.; Chu, N. J.; Petrache, H. I.; TristramNagle, S. T.; Nagle, J. F. Biophys. J. 2005, 88, 2626.

(11) Prosser, R. S.; Hunt, S. A.; DiNatale, J. A.; Vold, R. R. J. Am. Chem. Soc. 1996, 118, 269.

(12) Tan, C. B.; Fung, B. M.; Cho, G. J. J. Am. Chem. Soc. 2002, $124,11827$.

(13) Loudet, C.; Manet, S.; Gineste, S.; Oda, R.; Achard, M. F.; Dufourc, E. J. Biophys. J. 2007, 92, 3949.

(14) Park, S. H.; Loudet, C.; Marassi, F. M.; Dufourc, E. J.; Opella, S. J. J. Magn. Reson. 2008, 193, 133.

(15) Diller, A.; Loudet, C.; Aussenac, F.; Raffard, G.; Fournier, S.; Laguerre, M.; Grelard, A.; Opella, S. J.; Marassi, F. M.; Dufourc, E. J. Biochimie 2009, 91, 744. 
(16) Loudet, C.; Diller, A.; Grelard, A.; Oda, R.; Dufourc, E. J. Prog. Lipid Res. 2010, 49, 289.

(17) Raffard, G.; Steinbruckner, S.; Arnold, A.; Davis, J. H.; Dufourc, E. J. Langmuir 2000, 16, 7655.

(18) Arnold, A.; Labrot, T.; Oda, R; Dufourc, E. J. Biophys. J. 2002, 83, 2667.

(19) Riske, K. A.; Amaral, L. Q.; Lamy-Freund, M. T. Biochimica Et Biophysica Acta-Biomembranes 2001, 1511, 297.

(20) Wilkins, M. H. F.; Blaurock, A. E.; Engelman, D. M. Nature-New Biology 1971, 230, 72.

(21) Blaurock, A. E. Biochim. Biophys. Acta 1982, 650, 167.

(22) Nallet, F.; Laversanne, R.; Roux, D. Journal De Physique Ii 1993, $3,487$.

(23) Wiener, M. C.; White, S. H. Biophys. J. 1991, 59, 162.

(24) Nagle, J. F.; Tristram-Nagle, S. Biochim. Biophys. Acta 2000, 1469,159 .

(25) Pabst, G.; Kucerka, N.; Nieh, M. P.; Rheinstädter, M. C.; Katsaras, J. Chem. Phys. Lipids 2010, 163 (6), 460.

(26) Worthing, C. Biophys. J. 1969, 9, 222.

(27) Wiener, M. C.; Suter, R. M.; Nagle, J. F. Biophys. J. 1989, $55,315$.

(28) Zhang, R. T.; Tristram-Nagle, S.; Sun, W. J.; Headrick, R. L.; Irving, T. C.; Suter, R. M.; Nagle, J. F. Biophys. J. 1996, 70, 349.

(29) Pabst, G.; Rappolt, M.; Amenitsch, H.; Laggner, P. Phys. Rev. E 2000, 62, 4000.

(30) Sternin, E.; Nizza, D.; Gawrisch, K. Langmuir 2001, 17, 2610.

(31) Leonard, A.; Escrive, C.; Laguerre, M.; Pebay-Peyroula, E.; Neri, W.; Pott, T.; Katsaras, J.; Dufourc, E. J. Langmuir 2001, 17, 2019.

(32) Douliez, J. P.; Leonard, A.; Dufourc, E. J. J. Phys. Chem. 1996, 100,18450 . 